\title{
Three-dimensional plant modelling for remote sensing simulation studies using the Botanical Plant Modelling System
}

\author{
Philip Lewis \\ Remote Sensing Unit, Department of Geography, University College London, 26 Bedford Way, London WC1H 0AP, UK
}

(Received 10 November 1998; accepted 27 February 1999)

\begin{abstract}
A canopy reflectance model, the Botanical Plant Modelling System (BPMS), is described. The model is capable of ingesting information on plant structure from a variety of sources, such as stereo photogrammetric measurements, dynamic models and direct manual measurements, and creating three-dimensional (3D) models of the structure of individual plants in a canopy and/or underlying soil/topography. The canopy radiation regime is simulated from the definition of the illumination conditions, camera imaging properties and plant and soil radiometric attributes using Monte Carlo ray tracing. The model is aimed at simulating the canopy shortwave radiometric regime and its component parts to allow the study of the effect of plant geometry and other factors on the remote sensing signal. (C Inra/Elsevier, Paris.)
\end{abstract}

remote sensing / 3D modelling / Monte Carlo ray tracing / canopy reflectance modelling

Résumé - Utilisation du Botanical Plant Modelling System pour modéliser en 3D des plantes pour des études de simulation par télédétection. Un modèle de réflectance de couverts végétaux, le Botanical Plant Modelling System (BPMS), est présenté. Ce modèle permet de traiter des données sur la structure des plantes provenant de différentes sources, comme des mesures de stéréophotographie, des modèles dynamiques, ou bien des mesures manuelles directes, et permet de créer des modèles tridimensionnels de plantes individuelles et de former un couvert végétal disposé sur un modèle de sol. Les propriétés optiques du couvert végétal sont simulées par un algorithme de lancer de rayons basé sur la méthode de Monte Carlo à partir de la définition des conditions d'illumination, des propriétés optiques des capteurs et des caractéristiques radiométriques des plantes et du sol. Le modèle a pour but de simuler les différentes composantes du régime radiométrique des couverts végétaux dans les courtes longueurs d'onde pour permettre d'étudier les effets de la géométrie des plantes ainsi que des autres facteurs sur le signal radiométrique. (@ Inra/Elsevier, Paris.)

télédétection / modélisation 3D / lancer de rayon Monte Carlo / modélisation de la réflectance du couvert

Communicated by Gérard Guyot (Avignon, France)

Correspondence and reprints

E-mail: plewis@geog.ucl.ac.uk 


\section{Introduction}

The three-dimensional (3D) geometry of individual plants and their spatial arrangement in a canopy strongly affect the quantity and directional (angular) nature of radiation interception, scattering and emission by the canopy. Information on the structure of vegetation is therefore of critical importance to studies involving these quantities, such as remote sensing modelling and measurement.

In the field of remote sensing (and related areas concerned with radiation interception and scattering), a vast range of models to describe canopy reflectance have been developed. These can be broadly categorised into: turbid medium models; simple geometric models; hybrid (simple geometric bounding objects containing a turbid medium); and complex geometric models [17, 46]. Many of these models are aimed at an analytical approximation to canopy reflectance, allowing for rapid forward modelling (calculation of canopy reflectance given the canopy parameters) and some degree of understanding of the influence of the major factors affecting the remote sensing measurement. Such models are of great importance when estimating canopy variables from such measurements, whether directly attempting the inversion of a canopy reflectance model or studying the sensitivity of surrogate measures such as vegetation indices to these variables. In addition, they provide a clear, though generalised, statement of the way in which the various factors interact. The widespread availability of cheap, fast computers has, in recent years, lead to an increase in the complexity of the modelling tasks that can be undertaken for remote sensing simulation, and hence the type of question that can be addressed. Analytical solutions of radiation transport are still of great value, for the reasons outlined above, but it is now much more straightforward to consider numerical solutions for which the description of canopy geometry can be arbitrarily complex, and for which the range of assumptions required in modelling can be greatly reduced. Given a structural model (one composed of geometric primitives: triangular facets, paramet- ric surfaces, etc.) of an ensemble of plants in a canopy and some method of solving for radiative transport between surfaces for given viewing and illumination conditions, one can simulate the radiation regime of a canopy for a given set of radiometric attributes of the plant primitives. Using standard numerical methods such as Monte Carlo ray tracing (MCRT) [19, 33, 38, 43] or radiosity techniques $[9,18]$ for this task, a modelling environment can be formed requiring no major assumptions regarding the nature of the radiation interactions other than: i) they can be modelled by geometric optics; ii) a model of scattering exists to describe the interaction at each of the model primitives [28]. The models used need not describe all elements of a scene in a deterministic manner, as both ray tracing and radiosity techniques are applicable to simulating scattering by volumetric media, but in such cases one must note that additional approximations must be made with regard to the joint gap probability, clumping, etc., of a medium of finite scatterers.

With the flexibility offered by a numerical solution of radiative transport in mind, we can define a generic model of canopy reflectance which contains: i) a description of canopy geometry; ii) a statement of the plant component and soil scattering properties; iii) a description of illumination and viewing conditions; and iv) a solution for radiative transport in an arbitrarily complex environment. The Botanical Plant Modelling System (BPMS), which is described in this paper, is one expression of such a generic model. The initial ideas and implementation of the BPMS were presented by Lewis and Muller [32], with various developments of the model to date $[12,13,28,29,33,42]$. Applications of the model have been described [2, $3,6-8,10,14,20,30,31,34-36]$. This paper presents an overview of the BPMS and its role in remote sensing simulation.

Prusinkiewicz and Lindenmayer [40] describe 3D plant modelling using generative rules (Lindenmayer-systems or L-systems) in what they term a 'virtual laboratory'. In this laboratory (on the computer), one is able to experiment with potential rules which allow the generation of plantlike structures and examine the influence of poten- 
tial mechanisms on plant growth. Further developments in this field have allowed for exploring the interaction of these potential rules and environmental influences, such as light/space or water [37]. Similarly, AMAP [41] provides a framework for the expression of botanical development rules. The BPMS is designed to provide such a "virtual laboratory' for investigating the influence of plant and canopy structure on a remote sensing measurement of canopy reflectance.

Modelling of canopy reflectance within the BPMS is broken down into component parts comprising: i) a descriptive language of individual plant form; ii) canopy and topographic description; iii) plant and soil radiometric description; iv) creation of an explicit surface representation of the canopy and soil; v) methods of data capture/input; vi) description of sensor characteristics; vii) description of illumination conditions; viii) a radiation transport model. Section 2 provides a description of the model stages up to the point of the generation of an explicit surface representation of the canopy (i-iv). Section 3 covers methods of geometric data capture (v). Section 4 deals with the simulation of canopy reflectance and associated radiometric information (vi-viii). Section 5 discusses current and future applications of the work.

\section{BPMS modelling of canopy geometry}

\subsection{BPMS description of plant form}

\subsubsection{The BPMS as a form of L-system}

The plant modelling language of the BPMS is related to a specific form of L-system, a parametric L-system. Prusinkiewicz and Lindenmayer [40] provide a simple example of this concept with a tree generation model. The branches of the tree are considered as geometric primitives, in this case, cylinders with two geometric parameters, branch radius and branch length. Generative rules are applied within the L-system both to the creation of new primitives and to the modification of those primitive parameters, in this example, decreasing branch radius and increasing branch length with increasing branching order.

This concept is used in the BPMS, where flexible but simple representations of primitive forms are used as the basic unit of description of plant organs. Organ primitives currently implemented in the BPMS are: buds, stems, leaves and seed heads. Further compound primitives can be formed from these, for example in the creation of a compound leaf [28]. The core of the BPMS is a static description of plant/canopy state, but, as will be demonstrated, the links between the descriptive language and parameteric L-systems allow coupling to external dynamic models.

\subsubsection{Description of plant topology}

The arrangement of organs in a plant is described through the use of a bracketed string, as in bracketed L-systems. Unlike in traditional Lsystems, only the topological arrangement of the organs is usually given in the plant description 'word'. In the BPMS, a set of parameters is then associated with each of the organs, which is given after the word. The reason for this arrangement is that the organ parameterisation can be quite large, and can also contain comment statements, which would make for an illegible plant description. As an example of a plant topological description, figure 1 shows a plant-like form which can be represented by the following word (parsed from left to right ) $[b s b[+1][+1][+s b[+1][+1]][+s b[+1][+1]]$. The letters of the word are taken from the set $\mathrm{V}$ of letters: $\mathrm{V}=\{[],, \mathbf{b}, \mathbf{s}, \mathbf{l}, \mathbf{h}, \mathbf{+}\}$. The brackets [ and ] define a branching structure. Elements contained within the brackets are isolated from the rest of the string to the right. Letters $\mathbf{b}, \mathbf{s}, \mathbf{l}, \mathbf{h}$ represent the plant organs: bud, stem, leaf and seed head, respectively. Of these, $\mathbf{l}$ and $\mathbf{h}$ represent terminal primitives, i.e. no further primitives can be branched from them. The letter + represents a rotation primitive, a generalised description of the turtle rotations used in other L-systems. This is not strictly required in plant modelling as rotations can be performed within the organs, but it is sometimes convenient for a simple and clear plant description. By default, the occurrence of a primitive is uniquely identified by its position within the string (left to right), 


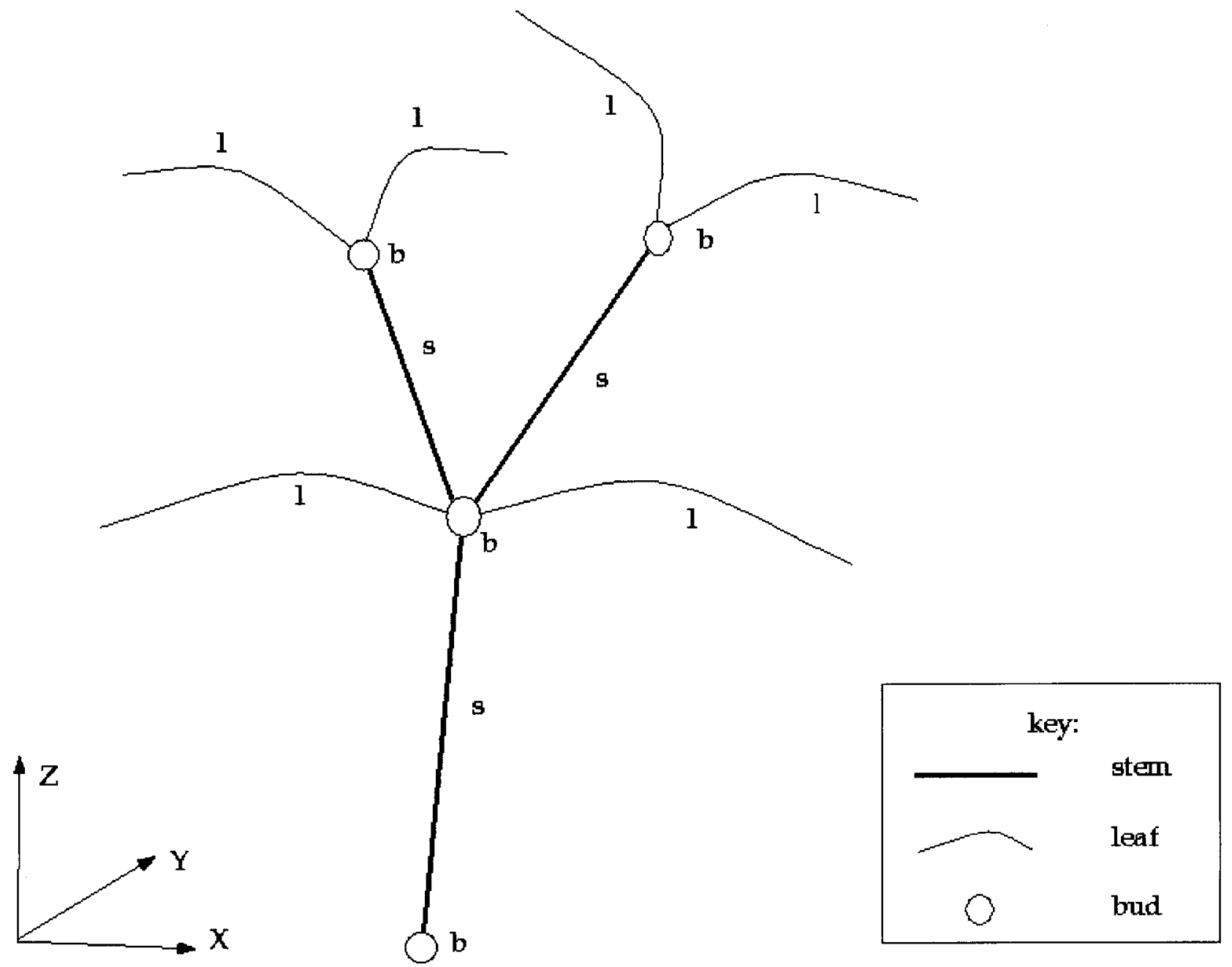

Figure 1. BPMS plant topology.

although a code attribute can also be associated with each letter, for example, l(1) labels an object I with the code 1 .

Each of the primitives $\mathbf{b}, \mathbf{s , l}, \mathbf{h}, \mathbf{h}+$ has a set of associated attributes. The attribute set for the bud primitive, for example, describe the material properties of the bud (typically the reflectance characteristics) and the dimensions of the primitive. A novel component of the BPMS is the parametric leaf form used for leaf, stem and seed head primitives. L-system models are generally defined through the defi- nition of 'turtle' movements in 3D space. Thus, by defining a series of commands such as 'move forwards', 'turn by an angle', the definition of $3 \mathrm{D}$ forms can be realised by the definition of such movements and rotations for some defined length step and set of local axes about which to rotate. The parametric model in the BPMS makes use of similar concepts except that functions are used to describe the rotation attributes, providing a continuous description. The definition of plant structure at a particular moment in time is achieved by a description of these functional attributes. 


\subsubsection{Plant organ geometry}

The attribute set for each primitive contains a definition of local rotational attributes, as well as material definition attributes (e.g. leaf reflectance), definitions of the variation in width of the primitive along its length (e.g. leaf width), and absolute (scaling) attributes, such as leaf length and leaf width. The angular parameterisation of the leaf (or stem or seed head) path in space is given as a triplet of Euler angles defining rotations about the axes of the local co-ordinate system on the leaf given as functions of normalised curvilinear distance from object base to tip, 1 . The local $\mathrm{x}$-vector is the leaf centre line normal vector in a downward-facing direction with respect to the leaf upper surface; the $y$-vector is across the leaf blade in the case of a flat leaf, but is more generally defined by the vector cross product of the $\mathrm{z}$ - and $\mathrm{x}$-vectors to lie in the leaf transverse section being normal to the $\mathrm{x}$-vector; the $\mathrm{z}$-vector defines the path of the leaf centre line, being the tangent vector of the leaf in the longitudinal direction. In the BPMS, these angles are defined as: twist, a rotation about the zvector; inclination, a rotation about the $y$-vector; and azimuth, a rotation about the $\mathrm{x}$-vector.

A partial set of attributes for a leaf primitive is given below. Attribute flags (names) are given in bold, followed on the same line by parameters associated with that attribute. As noted above, $I$ is the code for a leaf primitive, which is followed by a number $n$ associating this object with the nth occurrence of this primitive type in the topology description (overridden if a label is applied as described above). Parameters starting with a are absolute (effectively, scaling) parameters. Those starting with $f_{-}$are functional descriptions given over the interval $(0,1)$. Functional descriptions are followed by a code representing the type of function used to describe the variable, e.g. $n$ for a $n$ stage piecewise linear representation, $f$ for a Fourier series and $\mathrm{p}$ for a power series, allowing a relevant function form to be chosen for the parameterisation.
11
material
leaf
a_length
10.0

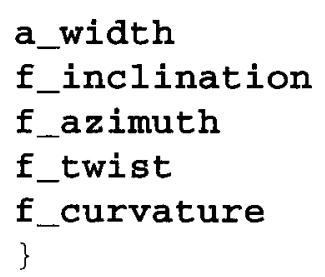

$\begin{array}{llllll} & 3.0 & & \\ n & 0 & 0 & 1 & 180 \\ n & 0 & 0 & 1 & 0 \\ n & 0 & 0 & 1 & 0 \\ n & 0 & 30 & 1 & 30\end{array}$

The format of the functional form for the $n$-stage piecewise linear functions used in this example is: $11, \mathrm{f} 1,12, \mathrm{f} 2, \ldots \ln , \mathrm{fn}$, where $\mathrm{li}$ is understood to mean the ith value of 1 in the description, with fi the corresponding value of the function being described. Thus, $n 001180$ describes a function which is 0 at the leaf base ( $1=0$, leaf insertion angle) and 180 (degrees) at the leaf tip $(1=1$, leaf tip angle), the function being linear in between. The parameter f_curvature describes the crosssectional curvature of the leaf, here constant at 30 degrees. A flat leaf blade would have a curvature angle of 0 degrees. A leaf which is fully rolled up would have a curvature angle of 180 degrees. The current implementation of the BPMS assumes the leaf cross-sectional form to be an arc of a circle, although this is readily changed to describe any leaf cross-sectional form. To demonstrate the use of the BPMS angular parameterisation in defining leaf form, the primitive given above is shown in figure 2, with the value of the leaf tip inclination varying from 0 to 180 degrees.

The BPMS, and most L-systems, use local rotational descriptors, an intrinsic method of form definition, as the plant can maintain the same form under global plant rotation and translation. Some L-systems, however, also include influences on turtle rotation defined in the global co-ordinate system, such as when modelling the influence of tropisms. Whilst one can, of course, translate between local and global rotational systems, it was felt that allowing a mixture of the two in the BPMS would be confusing for users. In addition, when attempting to derive a description of plant form from measurements, it would be impossible to decide which system to use. The plant organs can be considered as subject to local cellular growth and local stresses, even though they operate in an environment of global influences (light, gravity and airflow), so a local (intrinsic) system is probably the best compromise system for generic plant description. 


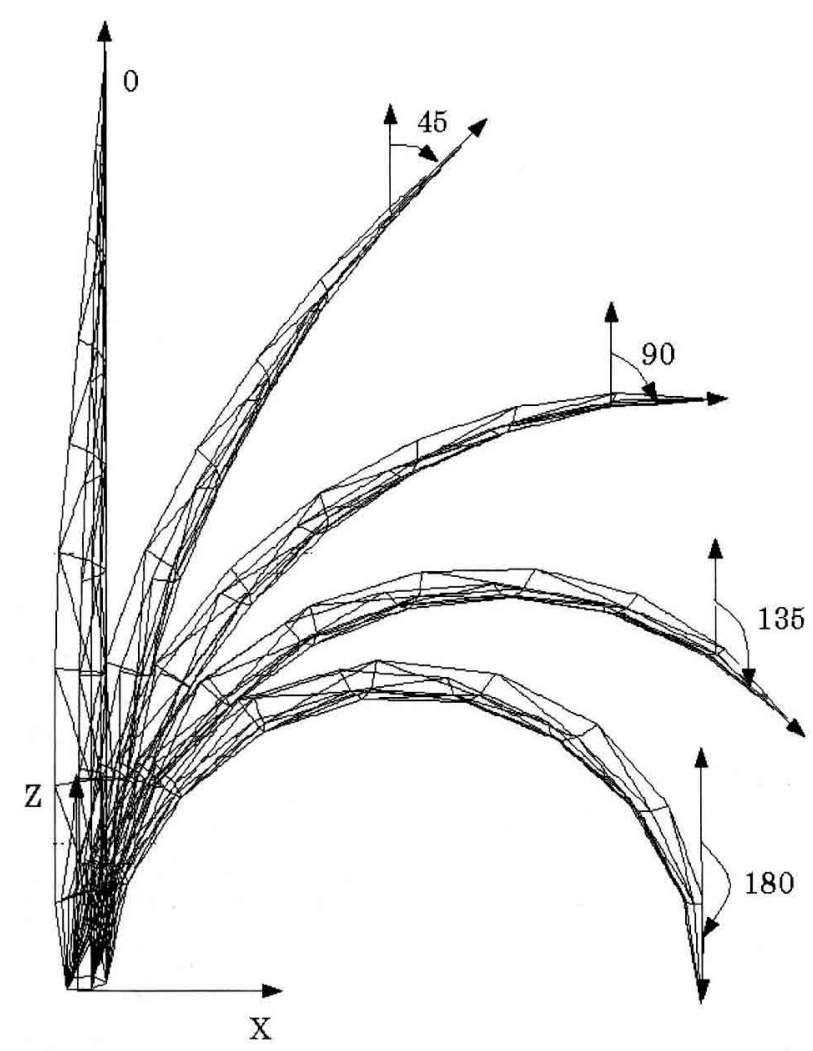

Figure 2. Variation of leaf tip inclination parameter.

However, if one were to attempt to model the state of otherwise identical plants grown under different illumination conditions, the (local co-ordinate system) BPMS description would be different for these different plants.

\subsubsection{Canopy description}

Given a definition of a number of plants, a canopy is formed in the BPMS by distributing the model ('clones' of the plants) over a field according to some defined planting pattern and probability of occurrence of each defined plant. This involves, for example, a definition of planting depth (the base of the plant), row direction and width, and linear plant density. The measures are typically defined by mean and standard deviations of these quantities, although any desired pattern of planting can be implemented. If a local height map defined as a digital elevation model (DEM) is available (e.g. describing soil (micro)topography), the plant heights are defined at each location relative to this. This can be used to define peaks and troughs of plant rows or more general topographic conditions.

Although the clones are allowed to rotate about their vertical axis according to some defined rotation function to increase the variability within the canopy, the use of clones in this way is a computational compromise, designed to keep down the requirements for computer memory usage. It also allows for the definition of a canopy given a limited number of measured plants. One problem with this is that it is difficult to avoid leaves from one plant sometimes intersecting with leaves from another plant in a cloned canopy. This could potentially be avoided if explicit representations are used for each plant, but intersection testing of this sort is not a solved problem, even in this case. The impact of this on the canopy radiation simulation is, at present, unknown, although it is likely to be greater for a dense canopy than for a sparse one.

Goel et al. [18] have implemented the concept of an 'infinite' canopy in his canopy model to avoid 'edge effects' at the limit of canopy definition, by replicating (effectively, cloning) a block of defined plants periodically in the horizontal direction. This is not directly implemented in the BPMS, as it provides unsatisfactory results for canopies which do not have a regular plant spacing. It is also impractical to use if there is any irregular topographic variation associated with the canopy. If it is required, it can be simulated using clones in the BPMS, for example, for simulations of canopies on a flat plane at high view zenith angles. More generally, it is necessary to define the canopy horizontal extent to be much greater than the area viewed by the sensor. Practically, this can be achieved by calculating the furthest extent of the canopy that might be viewed by a sensor and extending the canopy to three to four times this distance.

\subsubsection{Plant organ and soil material properties}

The parameter field material (2.1.3) associates a set of material attributes with each plant organ. Whilst these attributes can be very general and consist of any set of parameters we might want to associate with this organ (e.g. age, water status), the most typical, used for further processing to 
canopy reflectance, are spectral radiometric attributes. These attributes can be varied spatially over the extent of the organ. Each plant organ primitive has associated with it a local two dimensional coordinate system ( $\mathrm{U}$ and $\mathrm{V}$ ). The $\mathrm{U}$-co-ordinate is defined in the directional of normalised primitive length with limits 0 to 1 . The V-co-ordinate is, in the case of a leaf, defined across the leaf blade, i.e. normalised leaf width, from 0 to 1 . In the case of a stem or other primitive, similar concepts are used. If a spatially distributed definition of a material is used, the material name is associated in the material definition file with a material map. This map has associated with it a series of integer numbers which in turn point through a look-up table (LUT) to other materials, and a raster image made up of these integer numbers which provides the spatial distribution of the materials. Figure 3 shows an example of a material map applied to a leaf primitive to simulate variegation. In addition, a bump map [11] can be associated with an organ, again defined over the local two-dimensional co-ordinate system. A bump map describes high frequency but low magnitude surface perturbations, such as leaf vein networks. It is applied by altering the surface normal at a location on a primitive according to the perturbation defined at that location. Figure 4 shows an example of a leaf vein applied to the same leaf form used in figure 3.

The most common radiometric material, known in the BPMS as the 'standard reflectance material' (SRM) allows for the definition of a spectral function of primitive reflectance and transmittance for perfectly diffuse (Lambertian) and perfectly specular materials, although the latter is not often used in practice in canopy simulations. More typically, the SRM describes the Lambertian reflectance and transmittance factors as a function of wavelength. In addition, various anisotropic reflectance functions are implemented, principally, the bi-directional reflectance distribution (BRDF) models due to Pinty et al. [39] and Ahmad and Deering [1]. These models describe approximate analytical solutions for canopy and (in the case of the Ahmad and Deering model) soil BRDF and are mostly used to map a BRDF functions in terrain modelling applications, as performed by Burgess et al. [10] in

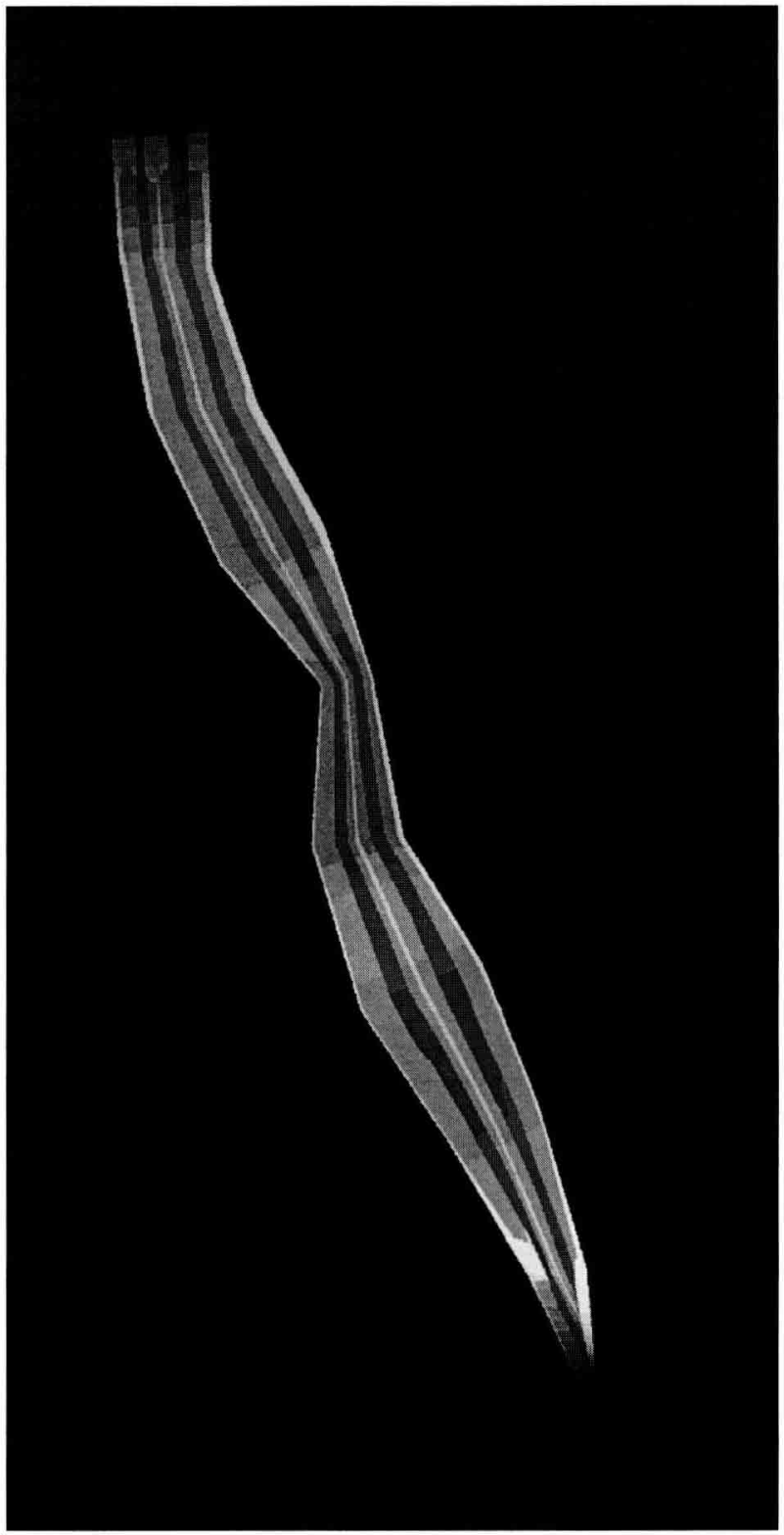

Figure 3. Variegated leaf modelled with a material map.

simulating the effect of rugged terrain on vegetation indices with ARARAT.

\subsubsection{Creation of an explicit canopy representation using geometric primitives}

Once a canopy has been described within the language of the BPMS as defined above, it must be 


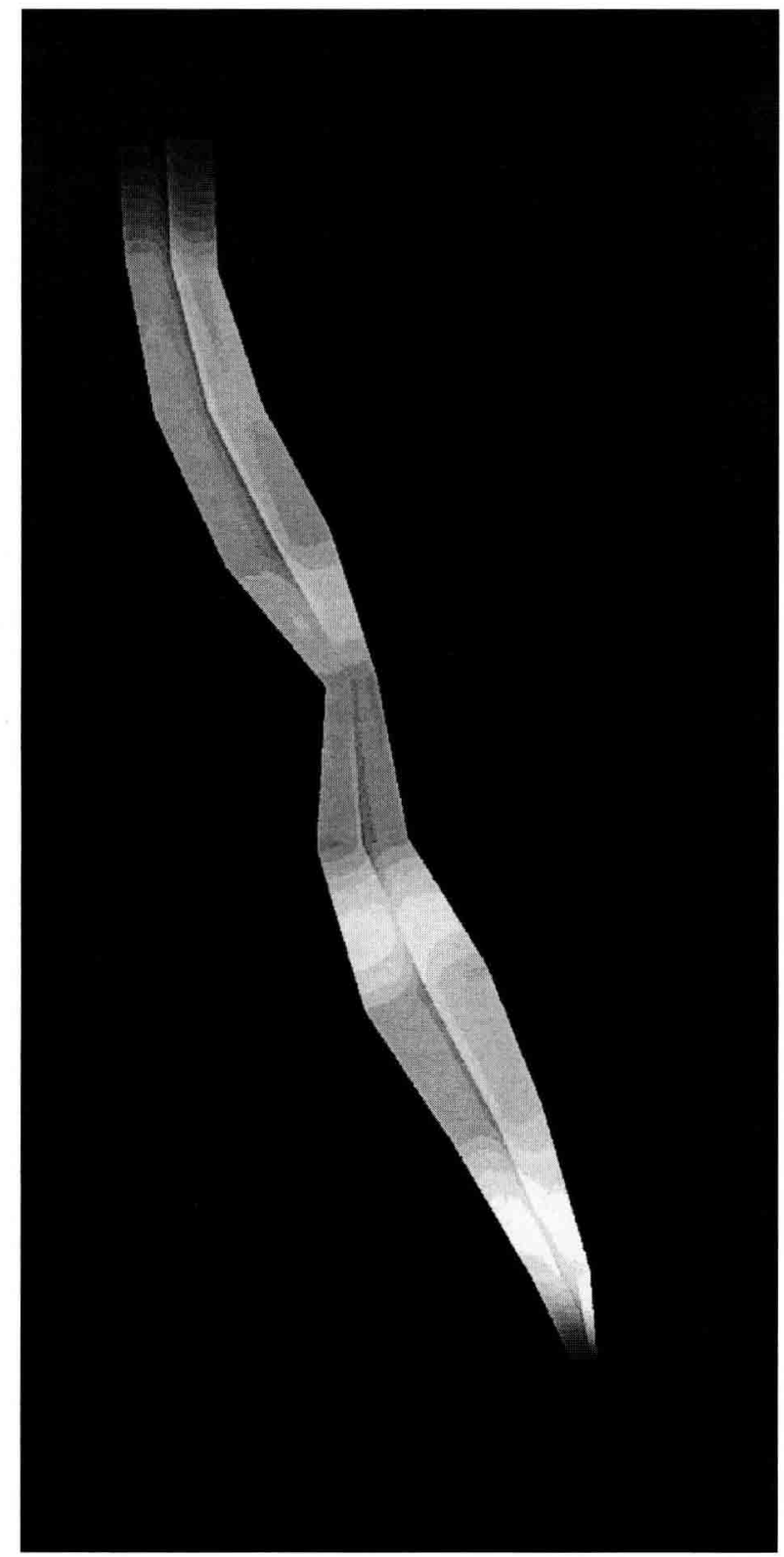

Figure 4. Leaf central vein modelled with a bump map.

converted to a form suitable for radiometric simulation. This involves parsing the topology string of each plant and generating explicit representations of the surface of each organ described. Leaf primitive descriptions are used to form a set of triangular facets or Bezier (bi-cubic) patches. Stem primitives are represented by a series of cylinders and spheres, bud primitives as spheres, and seed head primitives as cylinders and spheres.

During this process, various generalised canopy parameters can be calculated, such as the surface area associated with each defined material name, or their angular distribution. For example, if all leaf primitives are assigned the material name leaf, then the surface area and angular distribution results associated with this material type correspond to the canopy one-sided leaf area and canopy leaf angle distribution, the former of which can be easily converted to a measure of leaf area index (LAI) by dividing by the defined canopy areal extent.

Once a description of individual plants as a set of simple geometric primitives has been formed, it is straightforward to process these data to calculate other terms related to canopy radiometry, such as the leaf projection function ('G-function') and the leaf area scattering phase function.

\section{Plant measurement and data capture}

Plant structure information can be derived from a wide variety of sources for input to the model. The three main forms of input that have been used to date are: photogrammetric information; input from dynamic models; measurement of key plant parameters. The rest of this section will describe aspects of these different types of data inputs and present examples of ways in which they have be used in remote sensing studies.

\subsection{Photogrammetric data input and generalisation}

Photogrammetric measurement involves the acquisition of at least one pair of stereo images viewing a plant or number of plants. Typically, more than one set of stereo pairs is needed to view all plant organs. A set of well-distributed control targets is usually required in the images to allow reconstruction of the camera locations and orientations. 
Except in very sparse canopies, one encounters problems using photogrammetric techniques to measure individual plants in a canopy. These are mainly due to the overlap of the 'space' of neighbouring plants or the structural support given by neighbouring plants in a canopy. The use of photogrammetry implies that the plant surfaces one wishes to measure can be viewed from two or more locations, which is typically difficult or impossible to achieve for all leaves on a plant in situ in an undisturbed canopy. To try to get around this, various measurement strategies have been attempted within the BPMS and related work. These can be summarised as: i) extraction of individual plants from the canopy, as demonstrated in figure 5 which shows two pairs of stereo images of a wheat plant extracted from a nearby field and part of the photogrammetric control rig (targets) taken by Boissard and colleagues [6] at Inra Grignon, France; ii) removal of surrounding plants in the field to allow the viewing of individual plants, as performed by Lewis [28] to obtain maize geometric measurements; iii) destructive layered measurement, where a large area of the canopy is imaged at one time from above, as performed by Ivanov and colleagues at Inra Grignon, France [4, 21]. No single method is appropriate to all conditions: methods (i) and (ii) can result in a modification of plant geometry for dense canopies as it loses support from surrounding plants; methods (ii) and (iii) are subject to the prevailing weather conditions, most importantly wind (although wind shields can be used to ameliorate the situation); partial or complete destruction of the canopy in all methods means that the same plants cannot be followed for any multitemporal analysis.

Once a set of (single or multiple) stereo images has been obtained with suitable control target information, a camera model is constructed, giving the camera locations, attitude, etc., using digitised target locations [5]. Corresponding points on linear features (e.g. leaf centre lines) in the left and right images of a stereo pair are processed using the camera model to provide 3D vector data. For use in the BPMS, each vector is labelled to describe its position in the plant topology (e.g. tiller 1, leaf 1). Photogrammetric processing has been carried out using a number of photogrammetric packages [20, 28]. Such packages are not an intrinsic part of the BPMS, typically requiring filtering of the output format into a form usable by the model. This allows the user flexibility in defining a suitable package for measurement.

The 3D vector data derived from photogrammetric measurements are then processed within the BPMS to derive leaf length information and functional descriptions of leaf inclination and twist, etc. Information on the absolute organ parameters (leaf length, width, etc.) can also be extracted at this time, although it is sometimes more convenient to input these by some alternative method, such as by digitising relatively flat leaves on a flatbed digitiser or scanning the leaves to derive this information. External digitisation of leaf form can, in this way, provide more precise measurement of leaf dimensions and width functional variation and be associated with the $3 \mathrm{D}$ leaf vectors during subsequent processing. Once a functional BPMS description of all plant organs is obtained (figure 6) along with associated topological information, a description of an individual plant is stored (the wheat plant shown in figure 5 is reconstructed in this way in figure 7). Sampling of a number of plants (depending on the expected variability [21]) provides a sample set which can be used to investigate structural effects in a particular situation, but the description of each of these is rather complex, requiring hundreds of parameters.

The next stage of processing photogrammetrically derived data is to consider methods of generalisation. For example, once a set of functional forms of leaf inclination angle have been obtained, various parameterisations can be considered to simplify the description of form for a particular species. Whilst some progress has been made in this area (e.g. [20]), this aspect of the work within the BPMS must be considered to be still at a relatively early stage.

\subsection{Dynamic model data}

Although the BPMS description of a canopy is essentially static, it can be interfaced to dynamic 

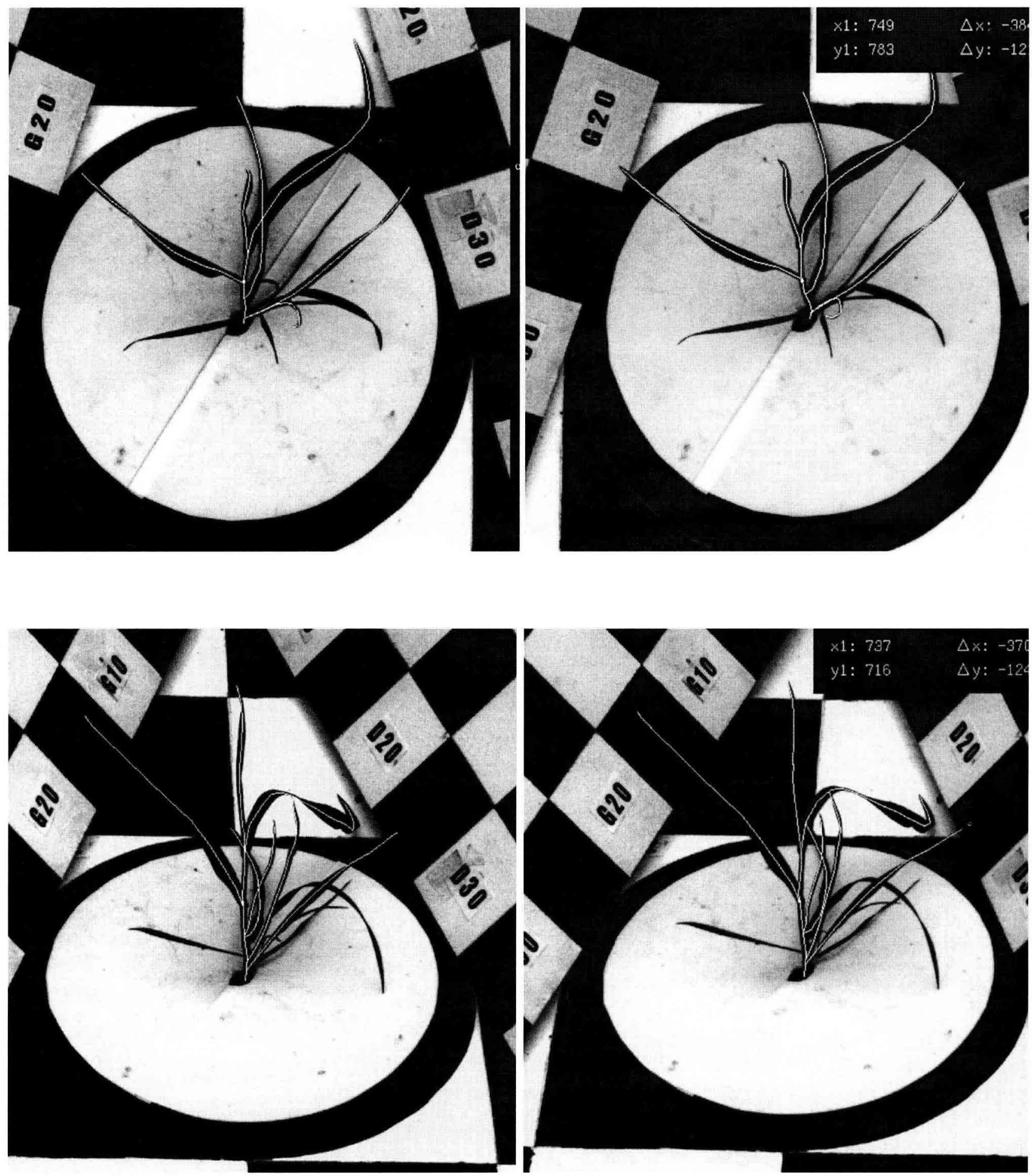

Figure 5. Laboratory stereo images of wheat plant: a) vertical view; b) oblique view. 

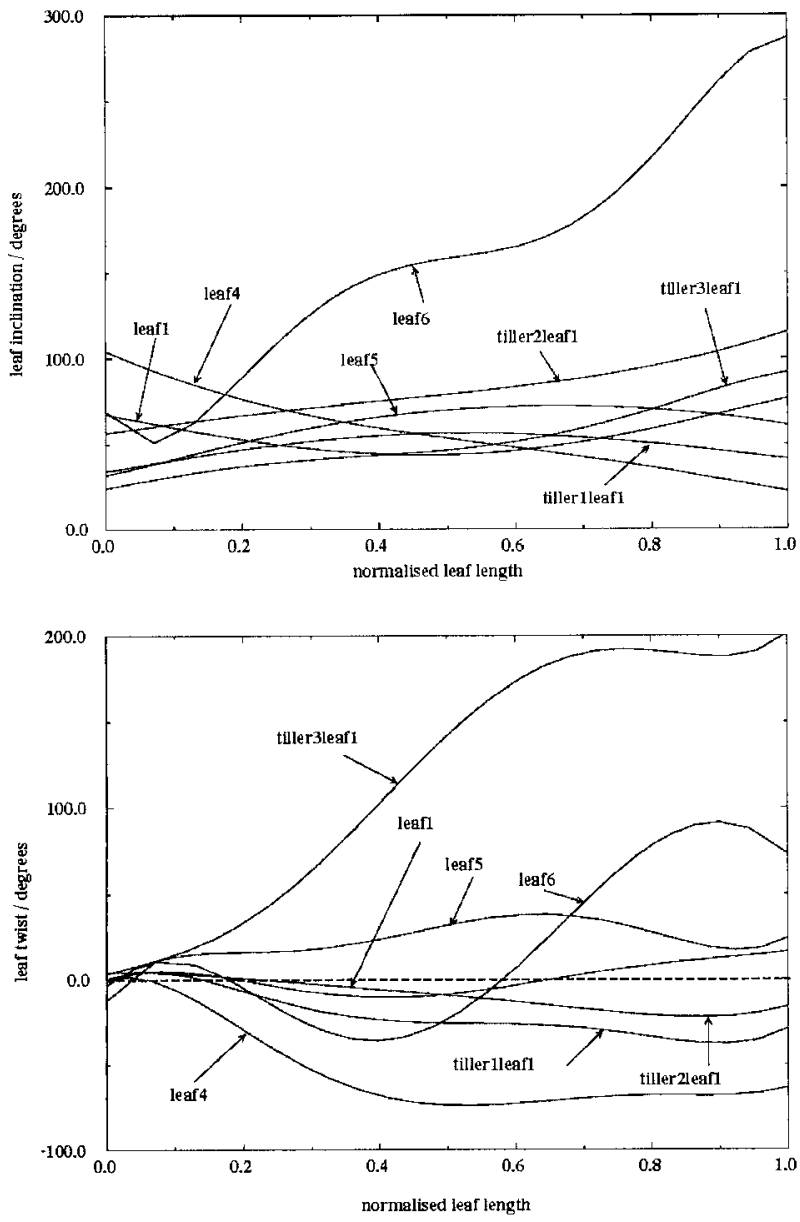

Figure 6. Leaf functional parameterisations of wheat plant (figure 5), a) Leaf inclination function; b) leaf twst function.

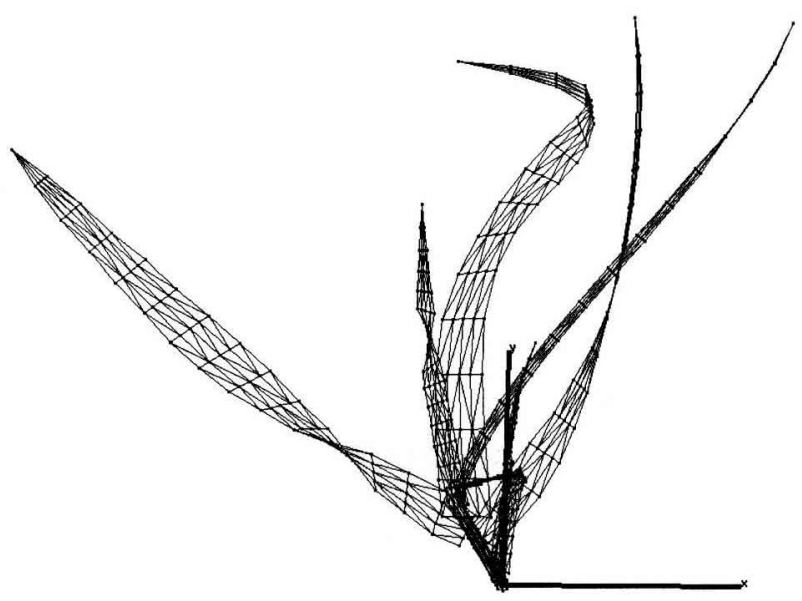

Figure 7. Reconstruction of wheat plant from functional representation shown in figure 6 . models. Two approaches to this have so far been taken with the BPMS: i) linking to a description from a parametric L-system of trees; and ii) creating a dynamic 3D model of wheat using the plant description language and simulation software of the BPMS. The first example, presented by Lewis [28], used a parametric L-system model of a tree defined by Prusinkiewicz and Lindenmayer [40]. For each iteration of the L-system, a plant description string was produced containing rotation primitives and branch primitives parameterised by their width and length. This description was readily translated into the BPMS description of a set of stem primitives for creation of a 3D surface model and subsequent radiometric simulation. The second example, described by Akkal [2] and Helbert [20] involved the collection of photogrammetric data of a large number of wheat plants. BPMS parameterisations were derived for each leaf of a sample set of plants, approximately every 2 weeks. The dynamic model, simblé, incorporates knowledge of the appearance of leaves and growth of tillers to provide a topological development model. The BPMS parameterisations of plant organ form were generalised and used to define stochastic growth rules for leaves and stems for a given variety of wheat at a given planting density as a function of integrated thermal time. The model has not yet been used for remote sensing simulations, but a form of validation was carried out using the BPMS. The dynamic model was run and LAI and canopy coverage calculated as a function of thermal time. These results were then compared with directly measured LAI/coverage data [2]. The results show that the dynamic model derived is able to reconstruct the structural relationships of the crop well. The measured results for very low LAI deviate slightly from the modelled values owing to inaccuracies in the field measurement of canopy coverage for low LAI.

\subsection{Measurement of key plant parameters}

If the functional form of the organs of a particular species is relatively straightforward, it is possible to arrive at an estimate of the parameters one would need for a generalised description of organ 
functional attributes without using detailed photogrammetric data. If this is the case, or if a photogrammetric study has already been carried out, then one may attempt alternative methods of deriving the required parameterisation for input into the BPMS. One way of doing this is to make manual measurements of, for example, leaf insertion and tip inclination angles, which may typically be measured with a protractor with perhaps 5 or 10 degrees error. These data are then used to parameterise the expected functional (leaf) form. In this way, one may conduct quite large studies of plant structure without the need for photogrammetric equipment; the weight of the effort is, however, altered from one requiring specific equipment and laboratory processing to one requiring a potentially large amount of manual measurement in the field.

A set of millet plants were manually measured as part of the HAPEX-Sahel field campaign in Niger in September 1992. Part of the purpose of the measurement was to use the models within the BPMS to compare with ground-based and airborne radiometric measurements [34]. The measurement of each millet plant took several hours for two operators owing to the complexity of the plant structure. Figure 8 gives some idea of the complexity of the canopy, showing a parcel of simulated millet plants from ground-level.

\section{The advanced radiometric ray tracer}

Once a geometric model of plants or a canopy has been described within the BPMS, a simulation of the radiation field outside (the remote sensing context) or within (the plant growth context) the canopy can be performed. As noted above, this involves the definition of a set of sensor characteristics, the description of a set of illumination conditions, and the use of a numerical radiation transport model.

\subsection{Camera definition}

Sensor characteristics, the camera location, orientation, optics and spectral sensitivity, are defined

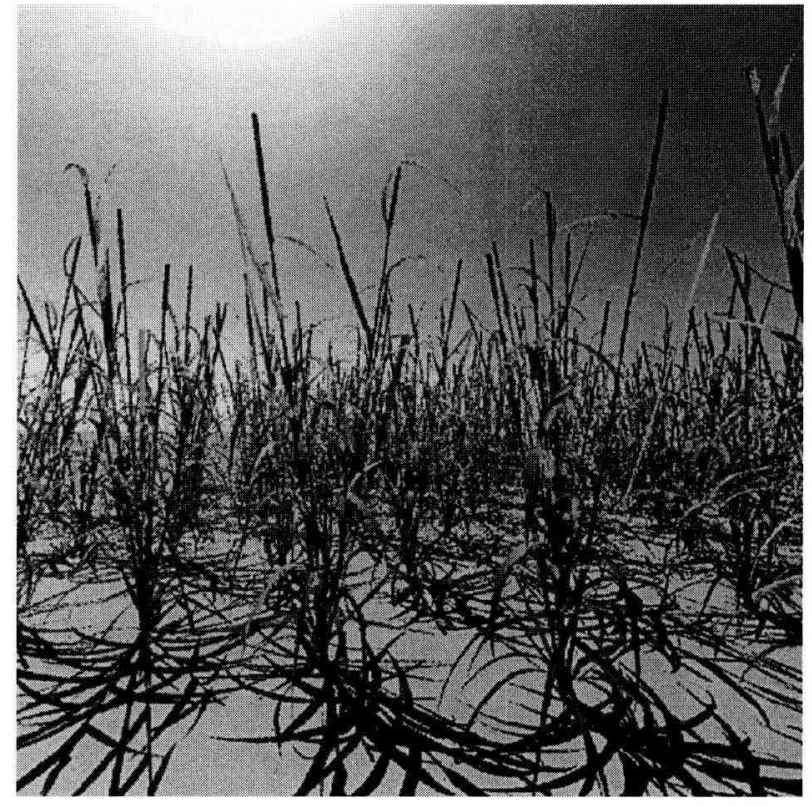

Figure 8. BPMS simulation of millet canopy.

through the use of a camera model. A variety of camera models are implemented in the BPMS as part of the ARARAT software. These are known as planar, spherical and albedo cameras. Additional cameras/scanning mechanisms can be added as required or created with external filters. For example, Burgess et al. [10] make use of the planar camera to define the response at individual pixels, but implement an external model to simulate the scanning mechanism and platform trajectory of the advanced very high resolution radiometer (AVHRR) satellite sensor.

\subsubsection{Planar camera model}

The most commonly used camera model is known as the planar camera. By default, this is a central perspective camera, the geometry of which is defined by a camera location (the location of the principal point on the imaging plane), a direction vector (the direction of the principal axis), and description of the focal length and pixel dimension. The latter two parameters can also be combined into a dimensionless zoom factor, the ratio of the focal length to the image semi-height ( zoom factor of 1 gives a field of view of 90 degrees). Often in 
directional reflectance simulation studies the user wishes to rotate a sensor on a 'virtual boom' about some point in the canopy at a given viewing zenith and azimuth angle, whilst viewing the same area of the canopy. This is achieved with a camera modelling program within the BPMS which alters the field of view of the sensor and the image dimensions to maintain a view of the same area of the canopy. For example, if a square image is defined for nadir viewing, the image aspect ratio (width divided by height) needs to increase (or the height decrease) with increasing view zenith angle, and the image field of view decreases, as shown in figure 9 to maintain the same viewing area. Figure 10

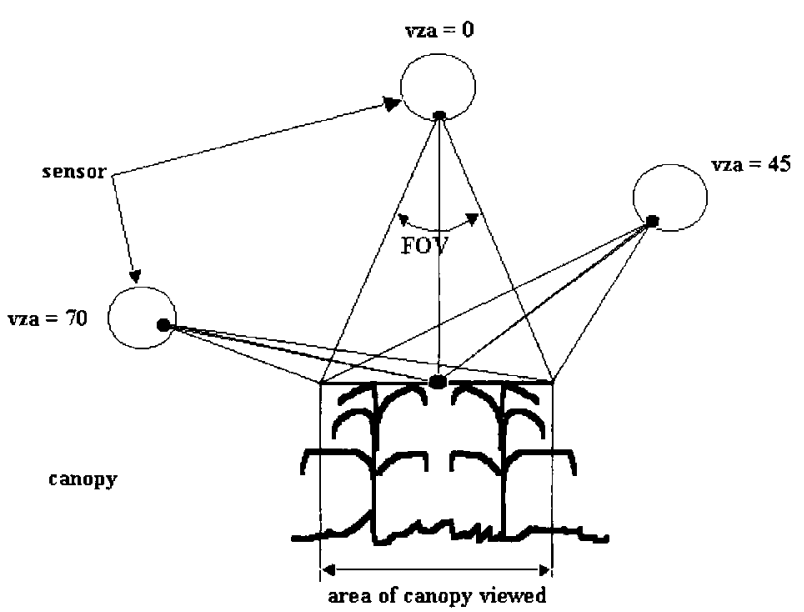

Figure 9. Variation in imaging parameters required to maintain the same canopy area. shows a set of simulations of a barley canopy using the BPMS with the changing aspect ratio of the camera allowing the same area of canopy to be viewed in all simulations.

By default, the camera is assumed to have an infinitesimal aperture, with a finite aperture being defined through the description of an $\mathrm{f}$-stop factor (the ratio of the focal length to the aperture diameter [28]). This feature is not typically used in radiometric simulations. When directional simulations are required, the planar camera can be specified to be orthographic, i.e. all photon trajectories to the camera are in the direction specified as the camera direction. This is the most typically used mode of camera operation.

\subsubsection{Spherical camera}

The 'spherical' camera has a hemispherical imaging surface, rather than the imaging plane of the camera defined above. This is useful for the calculation of gap probabilities and for looking at canopy projection and projected overlap functions. The camera location is taken to be the centre of the sphere. The viewing direction defines the orientation of the hemisphere. Most typically, this is oriented in the negative $\mathrm{z}$ direction, i.e. vertically downwards, providing an imaging surface over the upper canopy hemisphere. The radius of the hemisphere is defined as the camera focal length. As ARARAT uses reverse ray tracing (section 4.3.2) i.e. tracing of photon trajectories from the imaging surface through the sensor focal point, the default
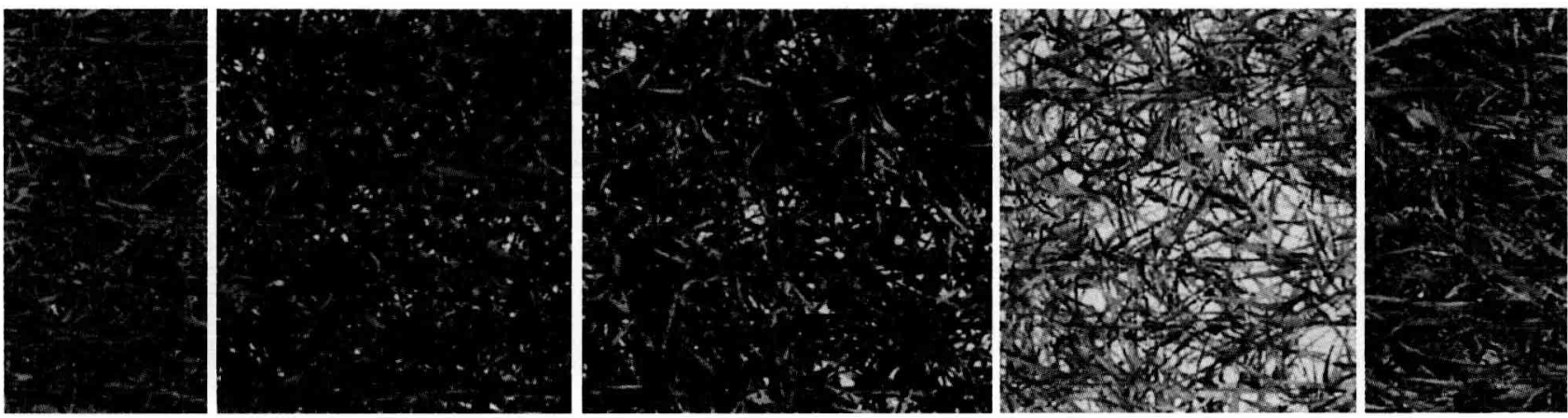

Figure 10. Barley canopy simulation demonstrating change in aspect ratio of image as view zenith angle is changed. View zenith angles of -60 to +60 degrees in steps of 30 degrees from left to right; solar zenith angle +30 degrees. 
mode of operation is for trajectories to be initiated from the camera surface towards the centre of the sphere. An option exists in the software to reverse the directions of the primary photon trajectories from the camera, i.e. to sample the paths from the focal point towards the imaging hemisphere. The spherical camera is then the equivalent of a 180 degree fisheye lens on a planar camera. Figure 11 provides an illustration of this for a millet canopy defined over a finite extent.

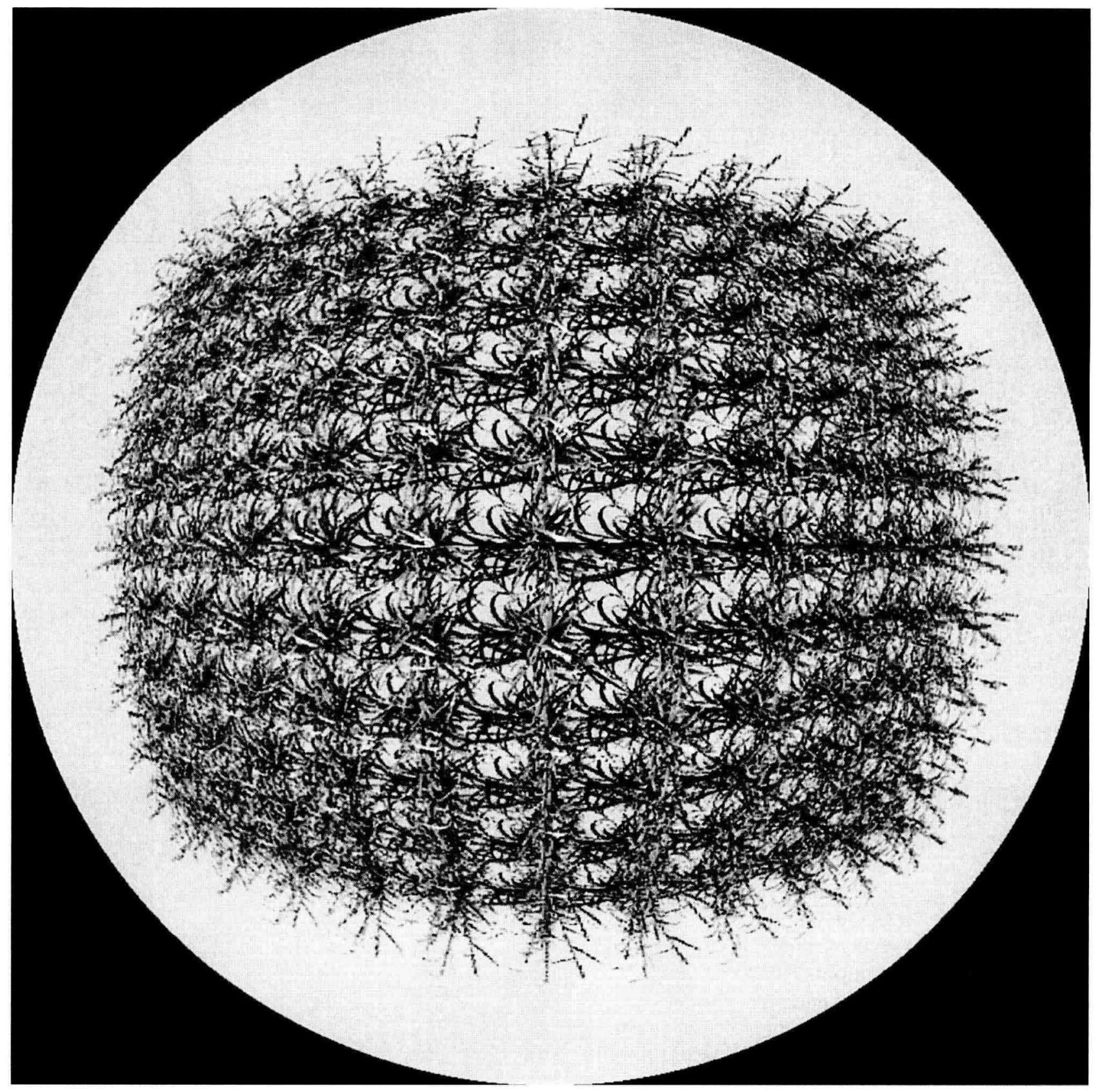

Figure 11. Spherical camera simulation - looking in on a millet canopy. 


\subsubsection{Albedo camera}

The albedo camera model is a variation of the hemispherical camera. The camera location and orientation are defined as previously, but the solid angle covered by each pixel in the output image is made to conform to a $\cos (q) \sin (q)$ weighting for a viewing zenith angle of $\mathrm{q}$. This weighting means that the mean pixel value over the image for a directional illumination source corresponds to the directional-hemispherical reflectance. As above, pinhole optics are assumed by default, but by defining the camera aperture to lie horizontally over some finite radius at the top of the camera allows for canopy directional-hemispherical reflectance to be calculated. Simulation of reflectance using an isotropic, diffuse illumination source provides a calculation of the bihemispherical canopy reflectance in the same way. If a combination of a directional illumination source and a sky radiance map (see below) are used in the simulation, the mean reflectance over the image provides a direct calculation of canopy albedo. Note that a canopy of infinite horizontal extent should be used in such a simulation. The use of this camera model is demonstrated in Lewis et al. [34] where measured canopy directional reflectance is used with a simple model to infer canopy directionalhemispherical reflectance. The BPMS is used to simulate directional-hemispherical reflectance of a millet canopy as a function of solar zenith angle to compare with the results of the simpler model.

\subsubsection{Sensor spectral response}

The spectral characteristics of a sensor can be defined in two main ways: i) a spectral response curve associated with a set of wavebands; ii) individual wavelengths. In the former case, ARARAT uses Monte Carlo sampling to sample in wavelength over the defined wavebands. In the latter case, simulations are performed at individual wavelengths, effectively impulse function wavebands. The definition of a set of bandpass functions is typically used if a simulation is being performed of a particular sensor system (e.g. AVHRR [10]), using measured functions or testing the applicability of new bandpass functions.

\subsection{Description of illumination conditions}

Illumination conditions in the BPMS are split into a direct component, representing direct solar radiation, and a diffuse component providing for scattered incident radiation. The simulation provides the user with top of canopy spectral radiance (for an external camera), for instance, in units of $\mathrm{Wm}^{-2} \mathrm{sr}^{-1} \mu \mathrm{m}^{-1}$, though this depends on the units used in defining the illumination conditions.

For a directional (orthographic, planar) camera with a point directional illumination source, canopy spectral bi-directional reflectance factors are calculated by ratioing the simulated radiance by the radiance under the same illumination conditions on a horizontal white Lambertian plane. Thus, a direct simulation of canopy spectral bidirectional reflectance factors can be achieved by illuminating the scene with a direct irradiance of $\mathrm{p} / \cos \left(\mathrm{q}_{\mathrm{s}}\right) \mathrm{Wm}^{-2} \mu \mathrm{m}^{-1}$, where $\mathrm{q}_{\mathrm{s}}$ is the illumination zenith angle. For the simulation of more realistic field conditions, a directionally varying sky radiance map is used. This can be derived from sky radiance measurements or from a model. A model derived from the analytical formulation of Zibordi and Voss [49] is included in the BPMS. An example of a modelled sky radiance distribution can be seen in the background of figure 8 . The sky radiance model is driven by a coefficient of ozone concentration and atmospheric precipitable water vapour, as well as parameters of the Angstrom formula for total aerosol optical thickness. It also requires coefficients of aerosol single scattering albedo, and parameters of a two-term HenyeyGreenstein function (to approximate the aerosol phase function). Height profiles of atmospheric constituents can also be provided so that the effects of altitude on the illumination field can be taken into account.

\subsection{ARARAT}

\subsubsection{ARARAT within the BPMS}

Figure 12 shows how the various components of the BPMS interact with the advanced radiometric ray tracer, ARARAT. This is the component of the 


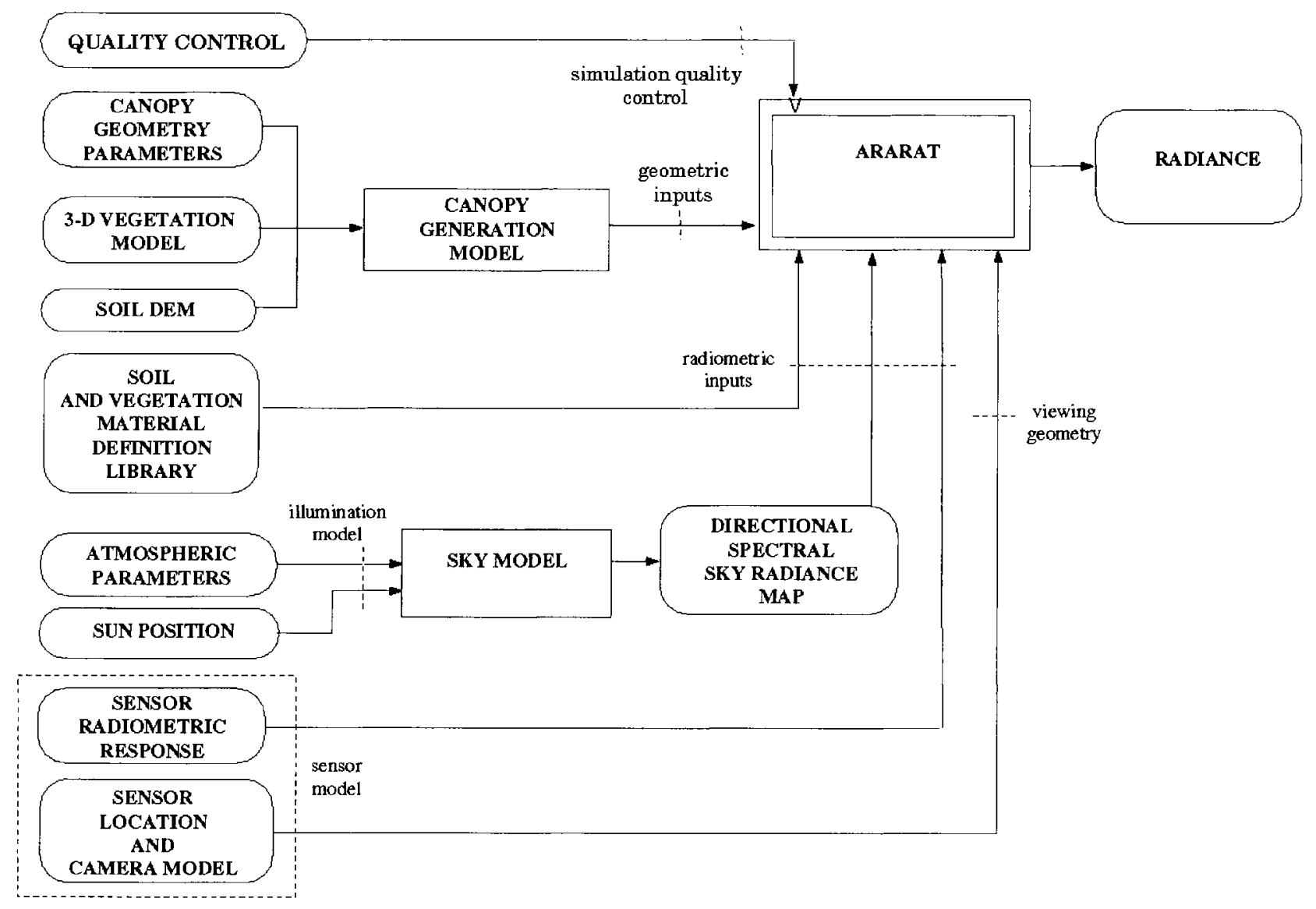

Figure 12. Flow diagram of BPMS.

model that performs radiometric simulation given the $3 \mathrm{D}$ canopy description (geometric model) and associated material, illumination (radiometric inputs) and camera descriptions (viewing geometry). In addition, a quality control can be set on the simulation. This controls the number of primary rays (rays fired from the imaging surface) used: the larger the number of rays, the lower the simulation noise, typically following an inverse root-square function. The quality of the simulation is also affected by the number of scattering interactions allowed in the simulation (in reverse ray tracing). A simulation of canopy radiance is produced in the units of the illumination model.

\subsubsection{Options for ray tracing}

Several options exist for ray tracing, the two basic types being forward and reverse ray tracing [16]. Govaerts [19] chooses to implement forward ray tracing in his canopy simulation model, whereby photon trajectories (rays) are traced from the illumination source, scattered or absorbed by objects within the scene, and collected by the imaging system (camera). When a ray intersects a scene object, the object material determines the probability of reflectance, absorptance or transmittance. A random number between 0 and 1 is generated and used to decide the fate of the photon. Since the probability of reflectance, etc., varies 
with wavelength, ray paths must be calculated individually for each waveband simulated. This approach is more easily understood as it is intuitive and in the direction of actual radiation transfer. The method is efficient if one requires a simulation over all upward directions from the canopy, but is seriously limiting if a simulation of a narrow field of view sensor is required, e.g. in the simulation of LiDAR (light detection and ranging) response. One particular advantage of forward ray tracing, however, is that it is straightforward to calculate both reflectance and absorptance by the canopy simultaneously.

ARARAT uses reverse ray tracing, whereby photon trajectories are traced from the viewer (camera) into the scene. If the ray intersects a scene object, sample rays are fired from the point of intersection to calculate the radiance incident on the object. One set of rays is fired towards the direction of the direct illumination field. Effectively, this determines whether or not the surface under consideration is in shadow, and so is known as a shadow ray. If the ray does not intersect with further objects along this path, the radiance on the object at the initial point of intersection is taken to be (for a Lambertian surface) the dot product of the local normal vector and the ray direction multiplied by the material reflectance factor. If the shadow ray intersects with another scene object on its way to the illumination source, further rays are fired until the source is reached and the radiation along that path attenuated by transmission through the intersected objects. In addition, diffuse sampling rays are fired from the point of intersection to calculate the radiance on the surface due to this component according to the sampling scheme of Ward et al. [48]. If the number of diffuse sampling rays fired at each intersection is set to 1 , a technique known as path tracing is performed, which Kajiya [24] claims to be efficient in terms of variance (noise) reduction as it maximises the proportion of primary rays which have the greatest contribution to the radiance being simulated. Govaerts [19] presents formulae similar to those of Ward et al. [48] for sampling a Gaussian specular function. Similarly, Shirley and Wang [44] present an analytical formula for Phong-like specular reflectance. The current implementation of ARARAT only deals efficiently with sampling a diffuse reflectance function, with non-Lambertian reflectance functions being implemented via an attenuation relative to the Lambertian component. Such a scheme is inefficient for reflectance functions which depart strongly from this model, although one advantage of the scheme is that multispectral ray tracing can be carried out efficiently by using ray bundles [28] whereby the same path through the canopy is used for all wavebands simulated. The different wavebands are distinguished by their varying attenuation components. This is a useful feature of ARARAT, as simulation of a large number of wavebands is only slightly slower than simulation of a single waveband as the largest cost in processing is typically the complex tests for ray intersections with scene objects. A more sophisticated general sampling scheme than the Lambertian one used in ARARAT is given by Govaerts [19], using an acceptance-rejection scheme for reflectance functions stored in a LUT. The main problem with such a scheme is that the sampling scheme will typically be different for each waveband, meaning that rays of different wavelengths cannot be kept together in a bundle [28].

Various standard ray tracing schemes are implemented within ARARAT to ensure efficiency of calculation. These include the use of bounding boxes around plant primitives and sub-primitives [16] so that if a ray does not intersect a bounding box, there is no need to test for intersection with the contents of the box; local plane sets [23], where objects are stored in order of occurrence along the principal co-ordinate axes to ensure efficiency of intersection testing; normal vector interpolation [45], whereby normal vectors calculated from the BPMS description of leaves are output for the vertices of each triangular facet making up a leaf primitive so that a smooth variation in surface reflectance can be achieved with a relatively small number of facets per leaf; and ray tree truncation, whereby ray paths are only traced up to a given number of interactions. Other efficiency features, such as the use of clones, bump mapping and material mapping have been noted above. 
Kirk and Arvo [25] argue that ray tree truncation is likely to introduce a bias by eliminating a large number of small contributions, but inspite of this, the ray tree must practically be truncated at some finite depth for which this bias is small as the processing task grows to the power of scattering order. The effect of this truncation depends on the reflectance and transmittance of the canopy elements and the canopy 'roughness' - the lower the absorptance and the smoother the surface (the less dense the canopy), the smaller the number of interactions required in modelling. Ray tree depths of around $7-10$ are typically found to be suitable for most canopy simulations $[28,31]$. Before embarking on a set of radiance simulations, it is generally worthwhile investigating the rate of attenuation per scattering order in ARARAT, to find an appropriate level for truncation.

\subsubsection{Outputs from ARARAT}

When a radiometric simulation is performed in ARARAT, a range of additional information associated with this can also be simulated. These include:

i) Direct and diffuse components: because ARARAT performs reverse ray tracing, it is straightforward to keep track of the components of radiance which are of direct and diffuse origin. Thus, simulated canopy radiance or reflectance can also be split up into direct and diffuse components. If the sky radiance distribution is assumed constant (isotropic), then this information can be recombined to give the canopy radiance/reflectance as a function of the proportion of diffuse illumination, without the need for further calculation. In addition, if an orthographic camera model is used, the diffuse component of reflectance for an isotropic illumination function is equivalent to the directional-hemispherical reflectance of the canopy. If an albedo camera model is used, the direct term is also equivalent to the directional-hemispherical reflectance, and the diffuse term is the bihemispherical reflectance.

ii) Radiance as a function of scattering order: the canopy radiance can be output in a form which gives the contributions to this term as a function of scattering order. Note that this information is also split into direct and diffuse components. This is useful for a variety of purposes, such as analysing the contribution of multiple-scattered radiation to the signal or examining the effects of ray tree truncation. Lewis and Disney [31] analyse the components of reflectance as a function of scattering order from a BPMS simulation of a barley canopy. These data are used to explore a formulation for multiple scattering in a barley canopy and to investigate the effects of row azimuth on this component of the radiation field.

iii) Proportion of sunlit and shaded components: ARARAT outputs the proportion of each material type (section 2.1.5) encountered at the first-order scattering level. This information is broken down into the proportion of each material in cast shadow or direct illumination. This information can be put to a variety of uses. For instance, Lewis and Disney [30] simulate components of reflectance of a millet canopy in order to understand the relationship between these and the way in which they are modelled using linear kernel-driven canopy reflectance models. To achieve this, they split the single-scattered reflectance field into components arising from canopy and soil interaction using information on sunlit and shaded leaf and soil material proportions, and compare these components with the way in which they are modelled in the simpler canopy reflectance models. In addition, one can note that the total proportion of (sunlit and shaded) soil calculated in ARARAT is equivalent to the integral of the canopy gap probability over the vertical height of the canopy. The proportion of sunlit soil viewed is related to the two-way gap probability integral. Careful processing of data on the proportion of canopy elements viewed can provide information on other important canopy properties such as effective scattering phase functions and leaf projection functions [28].

iv) Distance-resolved response: ARARAT has been recently modified to perform LiDAR simulation, giving radiance or reflectance as a function of ray path length (penetration depth into the canopy) $[12,42]$. The simulation proceeds in much the same way as the model of Govaerts [19], except that ARARAT performs backward ray tracing which is more efficient for this particular opera- 
tion. The behaviour of ARARAT is modified to account for the simulation of an active sensor in LiDAR mode. Rays are fired from the imaging plane (an orthographic camera) and scattered by the scene objects. Sampling of both the direct and diffuse components of the radiation field is considered at each level of interaction, but only radiation which originates from the illuminated area of the direct source (the LiDAR beam) contributes to the signal. Thus, if a (direct) ray trajectory exits the top of the canopy, a test is applied to see if the ray hits the LiDAR imaging plane. If it does, then it contributes to the LiDAR signal. If not, it is not considered to be within the illuminated area.

When operating in LiDAR mode, ARARAT outputs the components of the radiation field as a function of waveband and scattering order as before, but the signal is also broken down into distance-resolved histogram bins, according to the distance along the ray tree of the interaction. Figure 13 a provides an example of LiDAR distance-resolved output. It shows the contribution to single scattered reflectance from a millet canopy as a function of two-way (down and up) distance. The sensor is simulated at $3 \mathrm{~m}$ above the ground, with $0^{\circ}$ view zenith angle. The millet canopy height is around $2.25 \mathrm{~m}$. The response from the soil, occurring at $6 \mathrm{~m}$ (two-way distance) is not shown in figure $13 a$ as it is of a much higher magnitude than the canopy response. In addition to the distanceresolved histogram of the simulation, ARARAT also outputs a range image, giving the distance from the sensor to the first point of interaction with the canopy (figure $13 b$ ). This can be useful in interpreting the LiDAR response data, as it allows the user to relate the various features in the response to parts of the canopy geometry. Figure $13 c$ shows an image of single-scattered reflectance in the near infrared from the canopy for the same LiDAR simulation. Since each pixel in this image corresponds to a specific distance to the sensor in the range image, the reflectance as a function of range can be more finely resolved for single scattered radiation than as presented in the histogram. LiDAR simulations within the BPMS have been investigated by Cole [12], looking at the potential information content of the LiDAR signal over crop canopies, and
Roberts [42], investigating the operation of airborne and spaceborne LiDAR sensors over forest canopies.

\subsubsection{Volumetric primitives}

Although not strictly defined as a component of the BPMS, ARARAT can be used to simulate scattering from volumetric primitives. A number of bounding primitives (spheroids, etc.) are defined within ARARAT to contain a volumetric scattering medium. The properties of this medium are defined through a density function and an angular distribution function in much the same way as the model of North [38]. The reflectance and transmittance properties of the volumetric scatterers are defined by assigning a material as in standard BPMS operation. When a ray intersects the bounding volume primitive, the path length required to pass through the primitive is calculated. The leaf angle distribution function is then sampled to provide an estimate of the leaf projection function $G$ in the direction of the incoming ray. A random number $R$ between 0 and 1 is then generated and converted to a path length $m$.

$$
m=\frac{-\ln (R)}{u_{l} G}
$$

where $u_{1}$ is the leaf area density. If $m$ is greater than the object minimum path length, the ray passes through the object, otherwise it is intercepted at distance $m$ from the initial bounding volume intersection point. Direct and diffuse sampling rays are fired from an intersection point as above, but if the direction of illumination is close to the path of the original ray, some account must be taken of the increased probability of escape in this direction. In this case, the exponential joint gap probability model of Kuusk [27] is used to reduce the probability of interception on exiting the medium. This involves the definition of a scatterer size. Whilst this model provides a convenient and fast way of accounting for this enhanced joint gap probability, the way in which it is currently implemented is rather unsatisfactory, as rays which pass through a volumetric medium and are then scattered by another object (e.g. the soil) cannot be easily dealt 

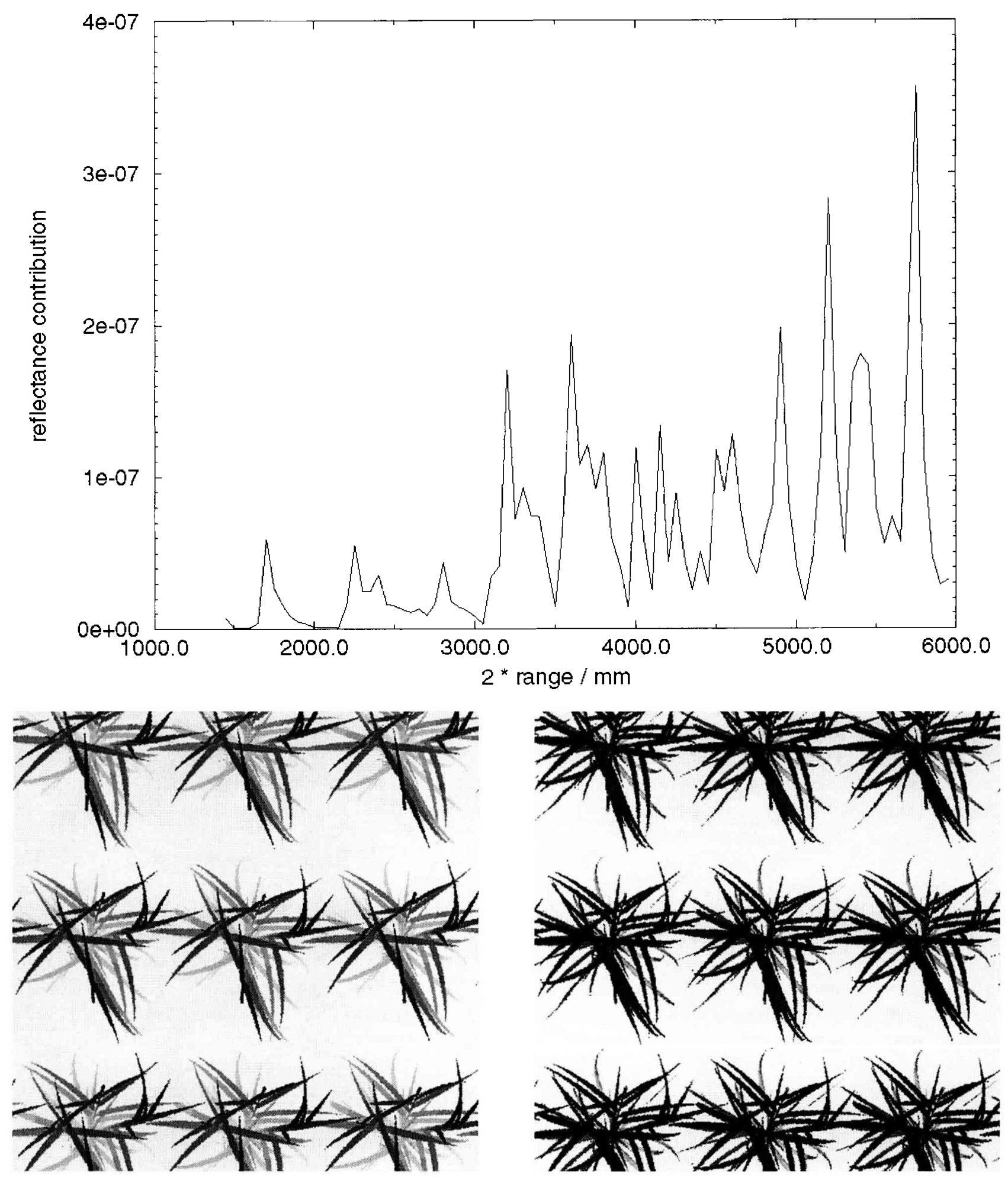

Figure 13. a) Distance resolved (LiDAR) response for near infrared for millet canopy (first-order scattering only). b) Relative penetration depth into the canopy (range image). c) First-order scattering LiDAR response viewed spatially for the millet canopy view. 
with in this way. Effectively, they 'forget' that they have travelled on a particular path through the canopy, and so have no enhanced probability of escape in travelling up the same direction. Thus, some proportion of rays which are transmitted through volumetric tree crowns and then scattered by the soil are incorrectly scattered by the crown on the way up through the canopy, reducing the effective soil reflectance contribution if the sensor is close to the retroreflection direction. Volumetric media are typically used within ARARAT to gain some idea of macrostructure effects on canopy reflectance for geometrically complex plants (e.g. trees) without the need for an explicit representation of each and every branch and leaf in each tree. This approach has also been successfully used by North [38] in simulating forest canopy reflectance.

When using volumetric media in ARARAT, the user must define a canopy geometric model, typically from a measured stem map or desired planting pattern, along with associated plant envelope characteristics. Cooksley [13] used volumetric primitives of this sort to investigate generalisation effects of canopy representation for a forest canopy by comparing a full structural representation with its volumetric equivalent. Roberts [42] used the same forest canopy model to investigate its LiDAR response. McDonald et al. [36] used volumetric representations of a forest canopy to investigate the sensitivity of vegetation indices to canopy cover. In this study the results of ARARAT were also compared with forest component information derived from a simpler forest canopy reflectance model. A key component of ARARAT in this work was its ability to accurately simulate multiple scattering in the canopy for the forest representation used allowing the impact of this on vegetation indices to be investigated.

\subsubsection{Topography}

ARARAT has also been used to simulate soil or topographic effects. Burgess et al. [10] used ARARAT to simulate topographic effects on satellite data over rugged terrain in New Zealand. A number of simulations were carried out, ranging from assuming a Lambertian reflectance function to the use of a directional reflectance function mapped onto the surface of the topography. In this way it was possible to simulate large areas of terrain without the need to produce models of individual plants over the area. The various tools within ARARAT also allowed topography-related components, such as the proportion of sky visible from each point on the terrain, to be calculated. In this way the study allowed for the various mechanisms by which rugged terrain can influence the signal received at a satellite to be quantified. Liang et al. [35] also made use of the topographic model within ARARAT in a comparison with various other rough surface models.

\section{Discussion and conclusions}

The BPMS is designed to model canopy spectral directional reflectance and associated radiometric information. The components of the model have been described in this paper along with a range of demonstration applications. In examining the use of such a model for remote sensing simulation studies it is worthwhile summarising these applications along with potential future applications of this and similar models. The primary remote sensing tasks that can be undertaken with $3 \mathrm{D}$ plant models are:

1) the development of an understanding of the influence of plant structure and arrangement on canopy bi-directional, directional-hemispherical and bi-hemispherical reflectance and absorptance;

2) the understanding of the impact of plant structure on components of the radiation field such as single- and multiple-scattered radiation; gap probability; sunlit and shaded components;

3) development and validation (benchmarking) of canopy reflectance and absorptance models through comparison with modelled canopy reflectance or components of the radiation field;

4) understanding correlations between canopy geometry parameters for specific cover types, e.g. relationships between LAI and canopy coverage, leaf angle distribution or leaf projection 
functions for a given crop at a given planting density and time;

5) the description of the dynamics of above by use of a dynamic 3D model;

6) the derivation of canopy model parameters through model inversion;

7) the calculation of spatial statistics of canopy reflectance.

The discussion of 3D models here is limited to those which use explicit surface representations rather than (bounded) stochastic descriptions. A number of sources of such (explicit) geometric models exist that have and might in the future be used for the above tasks. One can distinguish three main categories here: i) simple geometric forms; ii) those based on measurement of plant structure; and iii) those based on potential growth and environmental/mechanical response rules.

The work of Ross and Marshak [43] is illustrative of the former type of model. A simple plantlike form is generated as an explicit representation comprising a vertical cylinder representing the stem with oriented disks for leaves. Radiation transport is solved using MCRT. Such a model provides insight into the effects of structure on the radiation field (task 1) and could also be used to generate information relevant to task 2 . The relatively straightforward parameterisation also potentially lends itself to model inversion (task 6). It is not aimed at describing the response of a specific type of plant, but instead looking at more generic structural behaviour. A similar example is provided by the work of Borel et al. [9] where the canopy is described by an explicit representation of spatially distributed disks. There is no attempt here to mimic the form of plants in a canopy, but rather to gain an accurate simulation of the scattering from this simple canopy parameterisation by solving for radiation transport with the radiosity method. The BPMS has been used in a similar role for simulating forest macrostructure effects [10] and in examining generic issues in soil surface roughness [35].

The second type of model can be more specific as we can model some particular plant variety that we have measured and compare simulations based on these measurements to remotely sensed observations (e.g. using a different 3D model [4], or [34] using the BPMS).

The third type of model is well-represented by many approaches used within L-systems [10], whereby a typically simple set of generative rules are used to generate plant-like forms. These models can be used to explore more generic forms of complex structural effects, but the distinction between these models and the other models noted above is not always clear-cut, as the concept of Lsystems is generic enough to deal with all of them. As noted above, such models can also be used to explore environmental interactions given rules of how to respond to stimuli. The latter type of model is almost always dynamic, providing data on the development of structure over time, whereas the former types are typically static. A dynamic model based on plant measurement can be formed, linked to the BPMS [20] or other models [41] which can be of use in many of the categories outlined above. Measurements of plant structure are not used directly in a simulation but are instead used to calibrate the dynamics of the model either as a function of time steps [41] or as a function of integrated thermal time [20]. A dynamic model based on detailed measurement is often of more use than a static model as the dynamics of the canopy radiation and correlations between variables can be better explored. However, the measurement task for such modelling efforts is much larger, especially if one wishes to model plant dynamics under e.g. different illumination, planting density or soil conditions.

The application of explicit 3D modelling to tasks $1-5$ outlined above is relatively straightforward and is shown in current work in this area, at least for optical reflectance modelling. Applications noted in tasks 6-7 are areas in which $3 \mathrm{D}$ modelling could potentially have an impact in the future.

There has been little work on the inversion of complex 3D descriptions of plant form to date, although this fits in with the inversion philosophy of Knyazikhin et al. [26] whereby a look-up table is formed from an arbitrarily complex description 
of the canopy and an arbitrarily complex solution of radiation transport. A key issue here is the degree of specificity of a particular simulation and the amount of additional information that might be used in an inversion. In the general case where the crop variety, its history, planting pattern, etc., are unknown, there is little point attempting to invert some parameterisation of an extremely complex description of, say, a particular variety of crop. There will be insufficient information in the remote sensing signal to attempt this and too much coupling between parameters of the complex description (if this is anything approaching a parameterisation of individual leaf form and location). Inevitably, one is forced to use a much more generalised canopy description, although the model's generic tools for radiation transport could still be used for calculating the canopy reflectance. The more practical use of a model such as the BPMS in such a situation is to inform the modeller of the nature of the impact of specificity as a departure from the generalised description, and to gain an understanding of the relationship between derived generalised parameters and actual canopy parameters. If we are dealing with a much more limited situation where a good deal of detail is known about the canopy (variety, planting density, row orientation, etc.) then one might be able to make more direct use of a complex canopy description in inversion. The sort of role that the model might take in such a situation is to provide an expectation of canopy geometry, e.g. under unstressed conditions. If the canopy conforms to this expectation one might be able to derive leaf and soil radiometric properties from a radiometric simulation of the canopy. Equally, it might be possible to tell if the canopy departs from this expectation, as unrealistic values of canopy radiometric attributes would result. It is evident that dynamic canopy structural models would be required for such a task, and that the modeller would require a dynamic model parameterisation which was very specific to the canopy under consideration.

There has been relatively little work to date on the relationship between canopy characteristics and spatial and angular domain correlations between observations of the same canopy. Exceptions to this are the work of Jupp and colleagues [22] using simple geometric canopy reflectance models, and simulations using the 3D volumetric DART optical canopy reflectance model [15]. Sun and Ranson [47] investigate the spatial statistics of microwave data using a spatial model functionally similar to DART (in that a solution is formed over a 3D gridded (voxel) space). The vast majority of what work there is has concentrated on trees and bushes as the range of spatial dependencies of such canopies are more easily detectable with historical satellite remote sensing data with spatial resolutions of $10 \mathrm{~m}$ and below. A key element of this work, however, is the fact that an explicitly spatial model (though not necessarily one with explicit descriptions of surface primitives) was used to develop an understanding of and provide models of the relationships between canopy parameters and canopy spatial statistics. Although complex 3D models have not, to date, been used to investigate the nature of crop and other vegetation canopy spatial statistics, it is clear that the explicit spatial nature of the models provides the opportunity for such work.

The use of 3D plant modelling in remote sensing is still very much in its infancy but can be shown to have a great deal of practical use in developing this field of research and its applications. Key advances in other fields in recent years which have impacted on the use of such models for remote sensing simulation include: the vast increases in computer processing speed (and reduction in cost); advances in physically based methods in computer graphics, linking with earlier work in mathematics, physics and engineering to provide practical numerical methods for simulating radiation transport; the development of grammar systems for representing plant form and dynamics and the expression of botanical growth 'rules'; measurement technologies such as the availability of digital cameras for photogrammetry as well as the various other measurement devices such as 3D digitisers which can be used to measure plant form. Most of the requirements for the fruitful exploitation of this field of research are in place or rapidly near to being so. Whilst there has been considerable effort from a number of groups who have produced 
botanical growth rules for a relatively large number of tree species and other plants [41], it is clear that considerable progress is still required in characterising a sufficient range of plants under a sufficient range of conditions for more routine use in remote sensing simulations. Linked to this further progress still needs to be made in the development of efficient methods for the routine capture of plant structural data.

Acknowledgments: The author would like to thank the various University of London MSc Remote Sensing students who have experimented with the BPMS and related topics over the years. Thanks also to M. Disney, J.-P. Muller (UCL, UK) and M. Barnsley (UoW, Swansea, UK), P. Boissard, P. Valéry, N. Akkal, J. Helbert and J.-M. Meynard (Inra Grignon, France), for their contributions and discussions. Financial support has been provided by NERC (UK), and Inra (Fr.) for aspects of this work. The Remote Sensing Unit receives contributions for computer support and equipment from ULIRS. I would like to extend special thanks to B. Andrieu for organising the Inra workshop on 3D plant modelling in 1997 which provided the stimulus for finally writing this paper.

\section{References}

[1] Ahmad S.P., Deering D.W., A simple analytical function for bi-directional reflectance, J. Geophys. Res. 17 (1992) 18867-18886.

[2] Akkal N., Pilotage de la fertilisation azotée du blé d'hiver sur la base d'une évaluation précoce de la reflectance radiometrique ou du taux de couverture du sol, en vue d'une application a l'agriculture de precision, Ph.D. thesis, Inra, France, 1998.

[3] Akkal N., Boissard P., Chabanet C., Jeuffroy M.H., Lewis P., Meynard J.-M., Nitrogen fertilization management in winter wheat based on early estimate of the cover fraction, Proceedings 1 st European Conference on Precision Agriculture, Warwick University (UK), 7-10 September, 1997, pp. 405-412.

[4] Andrieu B., Ivanov N., Boissard P., Simulation of light interception from a maize canopy model constructed by stereo plotting, Agric. For. Meteorol. 75 (1995) 103-119.

[5] Atkinson K.B. (Ed.), Close Range Photogrammetry and Machine Vision, Whittles Publishing, Caithness, Scotland, 1996.
[6] Boissard P., Akkal N., Lewis P., Valery P., Meynard J.-M., Linking a 3D plant model database of crop structure to models of canopy development, Proceedings International Colloquium on Photosynthesis and Remote Sensing, Montpellier, France, 28-31 August, 1995.

[7] Boissard P., Akkal N., Lewis P., 3D plant modelling in agronomy, Association of Applied Biologists: Modelling in Applied Biology: Spatial Aspects, 24-27 June, Brunel, UK, 1996, pp. 125-129.

[8] Boissard P., Valery P., Akkal N., Helbert J., Lewis P., Paramétrisation d'un modèle architectural de blé au cours du tallage: estimation des paramètres de structure par photogrammétrie, in: Actes du Séminaire sur la Modélisation Architecturale, Département de Bioclimatologie Inra, Paris, 10-12 Mars, 1997, pp. 213-222.

[9] Borel C., Gerstl S.A.W., Powers B.J., The radiosity method in optical remote sensing of structured 3-D surfaces, Remote Sens. Environ. 36 (1991) 13-44.

[10] Burgess D.W., Lewis P., Muller J.-P., Topographic effects in AVHRR NDVI data, Remote Sens. Environ. 54 (1995) 223-232.

[11] Cabral B., Max N., Springmeyer R., Bi-directional reflectance functions from surface bump maps, Comput. Graphics (1987) 273-281.

[12] Cole M., LiDAR simulations over a crop canopy: an investigation of the waveform information content, M.Sc. thesis, University of London, 1998.

[13] Cooksley G., The importance of geometric structure in forest canopy reflectance modelling, M.Sc. thesis, University of London, 1997.

[14] Disney M., Lewis P., An investigation of how linear BRDF models deal with the complex scattering processes encountered in a real canopy, Proceedings IEEE Geoscience and Remote Sensing Symposium (IGARSS '98), Seattle, Washington, USA, 1998, CDROM.

[15] Gastellu-Etchegorry J.-P., Demarez V., Pinel V., Zagolski F., Modeling radiative transfer in heterogeneous 3 -D vegetation canopies, Remote Sens. Environ. 58 (1996) 131-156.

[16] Glassner A.S., An Introduction to Ray Tracing, Academic Press, 1989.

[17] Goel N.S., Models of vegetation canopy reflectance and their use in the estimation of biophysical parameters from reflectance data, Remote Sens. Rev. 4 (1988) 1-222. 
[18] Goel N.S., Rozenhal I., Thompson R.L., A computer graphics based model for scattering from objects of arbitrary shapes in the optical region, Remote Sens. Environ. 36 (1991) 73-104.

[19] Govaerts Y.M., A model of light scattering in three-dimensional plant canopies: a Monte Carlo ray tracing approach., Ph.D. thesis, Joint Research Centre, Ispra, Italy (Catalogue: CL-NA-16394-EN-C), 1996.

[20] Helbert J., Simulation du blé en 3D dans le but d'estimer l'interception du rayonnement, Stage de maîtrise de physique, Inra et université de Versailles Saint-Quentin-en-Yvelines, 1996.

[21] Ivanov N., Estimation de paramètres de structure du couvert végétal par stéréovision: application à l'étude de la pénétration du rayonnement, Ph.D. thesis, UFR de physique, université Denis-Diderot (Paris 7), France, 1994.

[22] Jupp D.L., Modelling directional variance and variograms using geo-optical models, J. Remote Sens. 1 (supplement: Special Issue of the First International Workshop on Multiangular Remote Sensing) (1997) 94-101.

[23] Kay T.L., Kajiya J.T, Ray tracing complex scenes, Comput. Graphics 20 (1986) 269-278.

[24] Kayiya J.T., The rendering equation, Comput. Graphics 20 (1986) 143-150.

[25] Kirk D.B., Arvo J.R., Unbiased sampling techniques for image synthesis, Comput. Graphics 25 (1991) 153-156.

[26] Knyazikhin Y., Martonchik J.V., Myneni R.B., Diner D., Running, S.W., Synergistic algorithm for estimating vegetation canopy leaf area index and fraction of absorbed photosynthetically active radiation from MODIS and MISR data, J. Geophys. Res. 103(D24) (1998) 32257-32275.

[27] Kuusk A., A computer efficient plant canopy reflectance model, Comput. Geosci. 22 (1996) 149-163.

[28] Lewis P., A botanical plant modelling system for remote sensing simulation studies, Ph.D., University of London, 1996.

[29] Lewis P., Boissard P., The use of 3D plant modelling and measurement in remote sensing, in: Proceedings VIIth International Symposium on Physical Measurements and Signatures in Remote Sensing, Courchevel, France, 7-11 April, 1997, pp. 319-326.

[30] Lewis P., Disney M., Examining BRDF model operation with the botanical plant modelling system, in: Proceedings Remote Sensing Society Conference, Reading, UK, 1997, pp. 298-303.
[31] Lewis P., Disney M., The Botanical Plant Modelling System (BPMS): a case study of multiple scattering in a barley canopy, in: Proceedings IEEE Geoscience and Remote Sensing Symposium (IGARSS '98), Seattle, Washington, USA, 1998, CD-ROM.

[32] Lewis P., Muller J.-P., Botanical plant modelling for remote sensing simulation studies, Proceedings IEEE Geoscience and Remote Sensing Symposium (IGARSS '90), Washington DC, USA, 1990, pp. 1739-1782.

[33] Lewis P., Muller J.-P., The Advanced Radiometric RAy Tracer: ARARAT, for plant canopy reflectance simulation, International Archives of Photogrammetry and Remote Sensing 29 Commission VII, 1992, pp. 26-34.

[34] Lewis P., Disney M., Barnsley M., Muller J.-P., Deriving albedo for HAPEX-Sahel from ASAS data using kernel-driven BRDF Models, Hydrol. Earth Syst. Sci. 3 (1999) 1-13.

[35] Liang S., Lewis P., Dubayah R., Qin W., Shirey D., Topographic effects on surface bi-directional reflectance scaling, J. Remote Sens. 1 (supplement: Special Issue of the First International Workshop on Multiangular Remote Sensing) (1997) 82-93.

[36] McDonald A.J., Gemmell F., Lewis P., Investigation of the utility of spectral vegetation indices for determining information on coniferous forests, Remote Sens. Environ. 66 (1998) 250-272.

[37] Mech R., Prusinkiewicz P., Visual models of plants interacting with their environment. Comput. Graphics 30 (1996) 397-410.

[38] North P., Three-dimensional forest light interaction model using a Monte Carlo method, IEEE Trans. Geosci. and Remote Sens. 34 (1996) 466-480.

[39| Pinty B., Verstraete M.M., Dickinson R.E., A physical model of the bi-directional reflectance of vegetation canopies 2: inversion and validation, J. Geophysic. Res. 95 (1990) 11767-11775.

[40] Prusinkiewicz P., Lindenmayer A., The Algorithmic Beauty of Plants, Springer-Verlag, New York, 1990.

[41] de Reffye Ph., Edelin C., Francon J., Jaeger M., Peuch C., Plant models faithful to botanical structure and development, Comput. Graphics 22 (1988) 151-158.

[42] Roberts G., Simulating the vegetation canopy LiDAR: an investigation of the waveform information content, M.Sc. thesis, University of London, 1998. 
[43] Ross J., Marshak A.L., Calculation of canopy directional reflectance using the Monte Carlo method, Remote Sens. Environ. 24 (1988) 213-225.

[44] Shirley P., Wang C., Distribution ray tracing: theory and practice, Proceedings Third Eurographics workshop on rendering, Bristol, UK, May, 1992, pp. 33-43.

[45] Snyder J.M., Barr A.H., Ray tracing complex models containing surface tessellations, Comput. Graphics 21 ( 1987) 119-128.

[46] Strahler A., Vegetation canopy reflectance modeling - recent developments and remote sensing perspectives. Proceedings VIth Colloquium on Physical
Measurements and Signatures in Remote Sensing, Val d'Isère, France, 17-21 January, 1994, pp. 593-600.

[47] Sun G., Ranson K.J., A three-dimensional radar backscatter model of forest canopies, IEEE Trans. Geosci. Remote Sens. 33 (1995) 372-382.

[48] Ward G.J., Rubenstein F.M., Clear R.D., A ray tracing solution for diffuse interreflection, Comput. Graphics 22 (1988) 85-92.

[49] Zibordi G., Voss K.J., Geometrical and spectral sky radiance: comparison between simulations and field measurements, Remote Sens. Environ. 27 (1989) 343-358. 